\title{
Activation of PI3 kinase/Akt/HIF-1 $\alpha$ pathway contributes to hypoxia-induced epithelial-mesenchymal transition and chemoresistance in hepatocellular carcinoma
}

\author{
MIN JIAO and KE-JUN NAN \\ Department of Oncology, the First Hospital of Xi'an Jiaotong University, Xi'an 710061, P.R. China
}

Received July 14, 2011; Accepted August 24, 2011

DOI: $10.3892 /$ ijo.2011.1197

\begin{abstract}
Hypoxia is known to promote malignant progression and to induce chemoresistance in cancer. However, the exact mechanisms driving hypoxia induced malignance remain elusive. We found that with exposure to hypoxic condition, hepatocellular carcinoma (HCC) cells experienced epithelialmesenchymal transition (EMT), with increased cell migration and invasion, and exhibited high resistance to chemotherapy. We demonstrated that hypoxia-induced EMT and chemoresistance were accompanied by increased HIF-1 $\alpha$ expression and activation of Akt. HIF-1 $\alpha$ could be blocked by PI3K inhibitor LY294002, indicating HIF-1 $\alpha$ activation was regulated by PI3K/ Akt pathway. Furthermore, we showed that inhibition of PI3K/ Akt and HIF-1 $\alpha$ enhanced the therapeutic efficacy of hypoxic chemotherapy in the HCC xenograft model. Our findings indicate that the activation of PI3K/Akt/HIF-1 $\alpha$ pathway plays a critical role in mediating hypoxia-induced EMT and drug resistance leading to unfavorable treatment outcome. Our study provides novel insights into the malignant progression triggered by hypoxic microenvironment in HCC cells.
\end{abstract}

\section{Introduction}

Hepatocellular carcinoma (HCC), an epithelial cancer originating from hepatocytes, is one of the five most common malignancies and the third most frequent cause of cancer deaths in the world $(1,2)$. The incidence of HCC has constantly increased in both Asian and Western countries in the last ten years (3). Long-term prognosis of patients with HCC is gener-

Correspondence to: Dr Ke-Jun Nan, Department of Oncology, the First Hospital of Xi'an Jiaotong University, Xi'an 710061, P.R. China E-mail: kejun.nan@gmail.com

Abbreviations: EMT, epithelial-mesenchymal transition; PI3K, phosphatidylinositol 3-kinase; HCC, hepatocellular carcinoma; HIF-1 $\alpha$, hypoxia induced factor 1 alpha; MDR1, multidrug resistance gene 1

Key words: hypoxia, hepatocellular carcinoma, epithelial-mesenchymal transition, chemoresistance, PI3K/Akt, HIF-1 $\alpha$ ally poor, and survival is mainly affected by the occurrence of metastases and chemoresistance (4). Currently, there is no effective systemic chemotherapy for this disease because tumor cells develop resistance to cytotoxic drugs such as cisplatin (5).

Hypoxia is a common feature of rapidly growing solid tumors, including liver cancer, breast cancer, prostate cancer and pancreatic cancer (6). Substantial evidence has shown that tumor hypoxia is an independent prognostic indicator of poor clinical outcome $(7,8)$. Hypoxic tumor microenvironment plays a prominent role in the development of malignant phenotypes, including local invasion, distant metastasis and chemoresistance (9-11).

Several studies have indicated that epithelial mesenchymal transition (EMT), a process vital for morphogenesis during embryonic development, is found in the most aggressive metastatic cancer (12). A hallmark of EMT is loss of epithelial cell markers, including the cell adhesion protein, E-cadherin, and acquisition of mesenchymal markers, such as vimentin and $\mathrm{N}$-cadherin. EMT is a key step leading to invasion and metastasis in solid tumors, including HCC (13). In ovarian and breast cancer cells, tumor hypoxia increases Snail levels and decreases E-cadherin expression $(14,15)$. These studies suggest a relationship between tumor hypoxia and EMT. It has been also proposed that the hypoxic tumor microenvironment plays an important role in the induction of chemoresistance (16). Multidrug resistance (MDR1) gene product P-glycoprotein (P-gp), an Mr 170,000 transmembrane protein associated with tumor resistance to chemotherapeutics, is also induced by hypoxia (17). However, the molecular mechanisms that relay the hypoxia signal into EMT and chemoresistance are still largely elusive.

In the present study, we demonstrated that hypoxia induce diverse molecular, phenotypic, and functional changes in HCC cells that are in line with EMT. Furthermore, we investigated the involvement of PI3K/Akt, HIF-1 $\alpha$, and MDR1 in hypoxiainduced cisplatin resistance in HCC. Finally, we evaluated the therapeutic efficacy of HIF-1 $\alpha$ blockade combined with chemotherapy in an in vivo nude mouse HCC model.

\section{Materials and methods}

Preparation of chemicals. The phosphatidylinositol 3-kinase (PI3K) inhibitor LY294002 was purchased from Cell Signaling 
Technology (CST, Danvers, MA, USA). The HIF-1 $\alpha$ inhibitor YC-1 and cisplatin were obtained from Sigma-Aldrich (Sigma, St. Louis, MO, USA).

Cell culture and hypoxic treatment. Human HCC cell line HepG2 (ATCC, Manassas, VA, USA) was maintained in highglucose DMEM (Invitrogen, Carlsbad, CA) supplemented with $10 \%$ fetal bovine serum (Life Technologies) and $100 \mathrm{mg} / \mathrm{ml}$ penicillin and $50 \mu \mathrm{g} / \mathrm{ml}$ streptomycin at $37^{\circ} \mathrm{C}$ in a humidified $5 \% \mathrm{CO}_{2}$ atmosphere. For hypoxic exposure, the cells of the logarithmic growth phase were placed in an anaerobic incubator (the oxygen concentration was adjusted to $1.0 \%$ with $5 \% \mathrm{CO}_{2}$ and $94 \% \mathrm{~N}_{2}$ ).

HIF-1 $\alpha$ siRNA transfection. Small interfering RNA (siRNA) against human HIF-1 $\alpha$ was purchased from Santa Cruz Biotechnology (Santa Cruz, CA, USA). HepG2 cells were seeded into a 6-well culture plate and allowed to reach 50-60\% confluency prior to transfection. Cells were transfected with HIF-1 $\alpha$ siRNA (75 nM) using Xfect transfection reagent (Clontech, Mountain View, CA) in hypoxia or normoxia as described in the text. Control cells were treated with transfection reagent and scramble siRNA only. All experiments were performed at least twice in triplicate.

Western blotting. Equal amounts of protein $(30 \mu \mathrm{g})$ were separated in $10 \%$ SDS-PAGE and transferred to nitrocellulose membrane. After blocking with 5\% non-fat dry milk in PBS containing $0.2 \%$ Tween-20, membranes were incubated at $4^{\circ} \mathrm{C}$ overnight with primary antibody, including primary antibodies against MDR1 (1:400), E-cadherin (1:200), N-cadherin (1:200), vimentin (1:1000), HIF-1 $\alpha$ (1:400), Akt (1:400), p-Akt (Ser473) (1:400) and $\beta$-actin (1:1000). All these primary antibodies were obtained from Santa Cruz. Membranes were then incubated with horseradish peroxidase-conjugated secondary antibodies (Sigma) for $2 \mathrm{~h}$. ECL reagent (GE Healthcare, NJ, USA) was used for protein detection.

In vitro migration and invasion assay. Cell migration was studied using $6.5 \mathrm{~mm}$ Transwell chambers with $8 \mu \mathrm{m}$ pores (Corning, NY, USA). Cells were trypsinized and resuspended in high-glucose DMEM with $0.5 \%$ bovine serum albumin. A total of $10^{5}$ cells in $0.3 \mathrm{ml}$ media were plated in the upper chambers in duplicate filters. DMEM medium with $10 \%$ FBS was used as a chemoattractant in the lower chamber. Cells were allowed to migrate in normoxia or hypoxia. After $12 \mathrm{~h}, 24 \mathrm{~h}$ and $48 \mathrm{~h}$, the non-migrating cells were removed from the upper surface of each Transwell by a cotton swab. Transwell membranes were then stained with crystal violet. Cells that migrated through the membrane to the lower surface were counted by light microscopy. To study cell invasion, the upper chambers were coated with ECM gel (BD Biosciences, USA). FBS was added to the lower chamber as described for the cell migration experiments. ECM invasion was allowed to progress for $12 \mathrm{~h}, 24 \mathrm{~h}$ and $48 \mathrm{~h}$ in normoxia or hypoxia. The ECM gel and cells on the top membrane surface were removed with a cotton swab. Penetration of cells to the underside of the membrane was determined as described for the cell migration experiments. All experiments were performed at least twice in triplicate.
Cell viability assay. Cell viability was determined using the 3-(4,5-dimethylthiazol-2-yl)-2,5-diphenyltetrazolium bromide (MTT) assay. HepG2 cells were seeded in triplicate at a concentration of $1 \times 10^{5}$ cells per well on a 96-well plate and cultured under normoxia. Twenty-four hours later cells were treated with different concentrations of cisplatin $(0,0.01,0.1,1,5,10,12.5,25$, 50 and $100 \mu \mathrm{M}$ ) under hypoxia or normoxia. After $48 \mathrm{~h}$, MTT assay was performed. MTT stock solution $(20 \mu \mathrm{l})(5 \mathrm{mg} / \mathrm{ml}$ in PBS, Sigma-Aldrich) was added to each well followed by incubation for $4 \mathrm{~h}$ at $37^{\circ} \mathrm{C}$. The formazan crystals were dissolved in $200 \mu 1$ of DMSO. Optical density values (OD) were determined at wavelength $570 \mathrm{~nm}$. Each assay was performed three times and the average results were calculated.

Cell apoptosis assay. HepG2 cells were seeded into $100 \mathrm{~mm}$ dishes and grown to $70 \%$ confluence. Then cells were treated with $20 \mu \mathrm{mol} / 1$ cisplatin for $48 \mathrm{~h}$ under hypoxia or normoxia. The cells were collected by digestion with EDTA-free trypsin. The cell pellet was washed with cold PBS twice and $1-5 \times 10^{5}$ cells were resuspended in $250 \mu \mathrm{l}$ Annexin $\mathrm{V}$ binding buffer $(10 \mathrm{mM}$ Hepes pH 7.4, $150 \mathrm{mM} \mathrm{NaCl}, 2.5 \mathrm{nM} \mathrm{CaCl}_{2}, 1 \mathrm{mM} \mathrm{MgCl}_{2}$ and 4\% BSA). Next, the cells were stained with Annexin V FITC and propidium iodide (PI) for $15 \mathrm{~min}$ in the dark and subjected to flow cytometry analysis within $1 \mathrm{~h}$.

Tumor growth in xenograft mice. For establishing a hepatocarcinoma xenograft tumor model, the viable HepG2 cells (2.0x $10^{6}$ cells in $0.2 \mathrm{ml}$ of serum-free DMEM medium) were injected subcutaneously into the flanks of 6-weeks old male $\mathrm{BALB} / \mathrm{c}$ nude mice. Tumor size was measured in three dimensions every two days, and the tumor volume was calculated by the formula: largest diameter x (smallest diameter) ${ }^{2}$ x 0.5 . Animal experiments were approved by the Institutional Animal Care and Use Committee and conducted in accordance with Institutional guidelines. When the tumors reached a mean size of about $200 \mathrm{~mm}^{3}$, the treatment was initiated. The sufficient dosage of cisplatin was determined by initial in vitro experiment. HepG2 xenograft $\mathrm{BALB} / \mathrm{c}$ nude mice were randomly separated into four groups (eight mice in each group, $n=8$ ): (a) twice weekly intraperitoneal (i.p.) injection of cisplatin (4 $\mathrm{mg} / \mathrm{kg}$ ) alone, (b) twice weekly i.p. injection of both cisplatin $(4 \mathrm{mg} / \mathrm{kg})$ and YC-1 $(30 \mathrm{mg} / \mathrm{kg})$, (c) twice weekly i.p. injection of both cisplatin $(4 \mathrm{mg} / \mathrm{kg}$ ) and LY294002 (75 mg/kg), and (d) twice weekly i.p. injection of DMSO as control. All groups were treated for 8 weeks. Tumor volume was calculated as in the initial experiment. Treated mice were closely monitored for any signs of progressive disease and sacrificed if they became moribund. Tumors were measured every two days.

Statistical analysis. All results are expressed as the mean \pm SEM. The data were analyzed using one-way factorial analysis of variance (ANOVA) with Student t-tests for comparisons between the means. The potential of cisplatin, YC-1 and LY294002 for inhibition of in vivo tumor growth was analyzed using Tukey's HSD test. The level of significance was set at the $\mathrm{P}<0.05$. In the survival model, the Kaplan-Meier method was used to analyze survival times and log-rank tests were done for the purpose of comparison. Statistical analysis was carried out using SPSS software (SPSS, Inc., Chicago, IL.) 
A

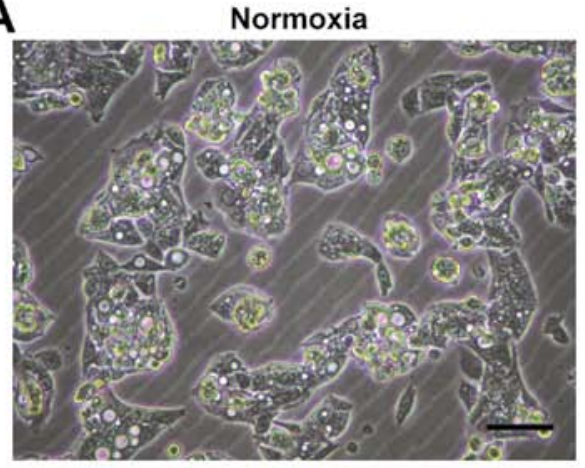

B

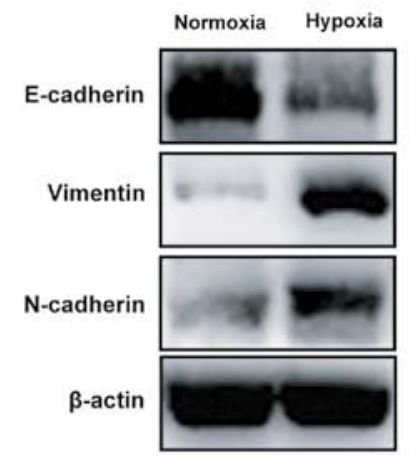

C

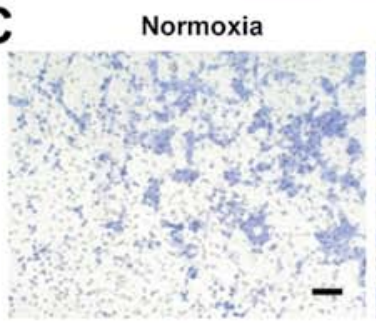

D

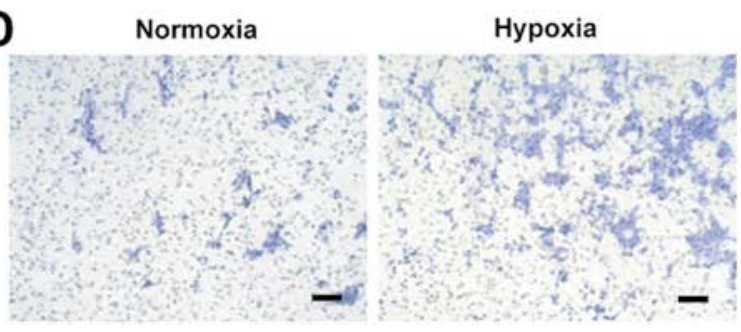

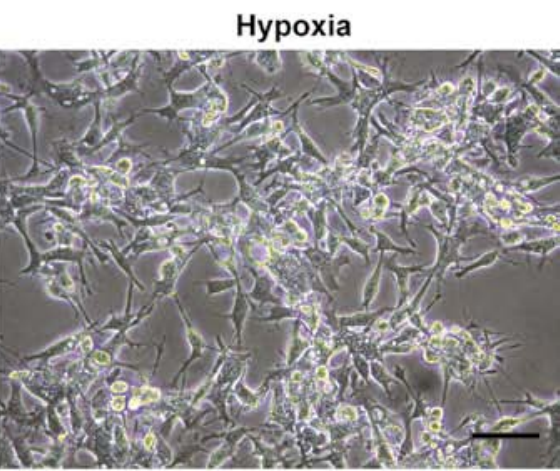

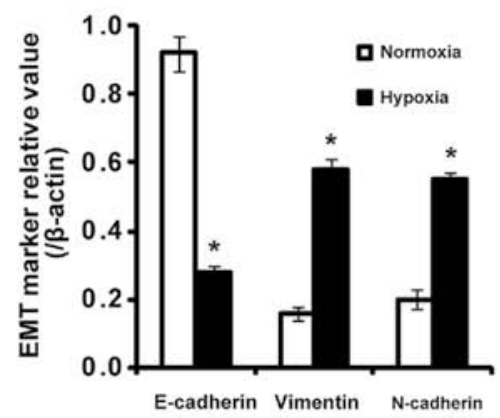

Hypoxia
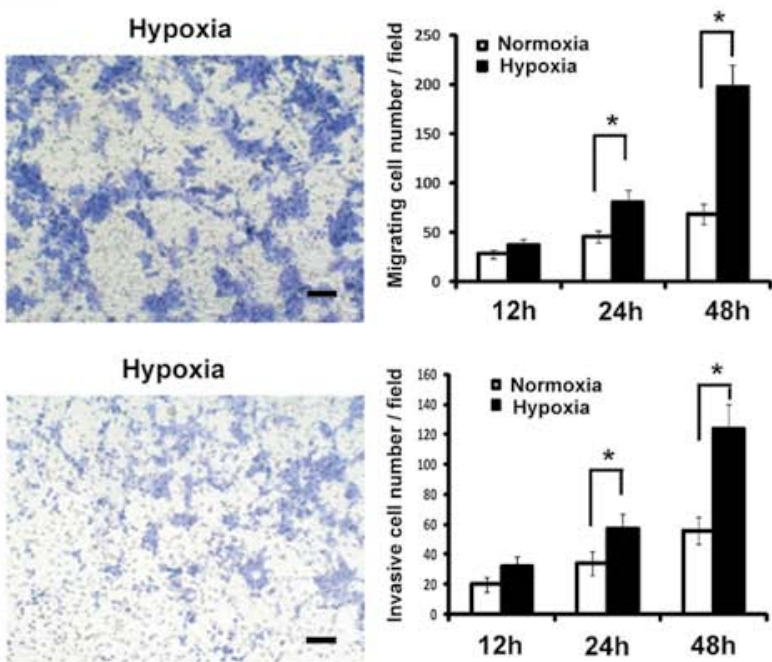

Figure 1. Hypoxia induces EMT in HepG2 cells. (A) Cells were cultured in $21 \% \mathrm{O}_{2}$ (normoxia; $\mathrm{N}$ ) or $1.0 \% \mathrm{O}_{2}$ (hypoxia; $\mathrm{H}$ ) for 48 h. The cell images were captured by phase-contrast microscopy. (B) EMT related marker, E-cadherin, vimentin and $\mathrm{N}$-cadherin, were examined by Western blotting. $\beta$-actin was used as internal control. The protein relative value ( $\beta$-actin) is plotted in the right panel (mean \pm SEM in three separate experiments). ${ }^{*} \mathrm{P}<0.05$ cells in hypoxia compared with in normoxia. The migration ability (C) and invasive ability (D) were analyzed by the migration and invasion assay. Representative images (48 h) are shown and the number of invasive and migrating cells per microscopic field was plotted (mean \pm SEM in three separate experiments). "P<0.05 cells in hypoxia compared with normoxia. Scale bar, $100 \mu \mathrm{m}$.

\section{Results}

Induction of EMT in HCC cells by hypoxia. Hypoxia-induced EMT transformation wasinvestigated by examining the morphologic changes, EMT-related marker and functional changes. We cultured $\mathrm{HepG} 2$ cells in normoxic $\left(21 \% \mathrm{O}_{2}\right)$ or hypoxic conditions $\left(1 \% \mathrm{O}_{2}\right)$. Firstly, we analyzed the cell plasticity. As shown in Fig. 1A, after a $48 \mathrm{~h}$ hypoxia exposure, HepG2 cells, which typically look like epithelia with well-developed cell junctions, acquired a spindle shape and generally lost cell-cell contact. To confirm the EMT transformation, we assessed the expression of EMT-related markers, such as E-cadherin, vimentin and $\mathrm{N}$-cadherin. As shown in Fig. 1B, after $48 \mathrm{~h}$ in hypoxia, the
E-cadherin protein level significantly decreased when compared with that in normoxia $(\mathrm{P}<0.05)$. However, the vimentin and $\mathrm{N}$-cadherin level was significantly higher in hypoxia than that in normoxia $(\mathrm{P}<0.05)$. EMT is thought to promote cancer cell migration and invasion. Therefore, the cell migration and invasion ability were analyzed using a Transwell chamber. As shown in Fig. 1C, migration was increased 1.47 \pm 0.32 -fold at $24 \mathrm{~h}$ and $2.36 \pm 0.12$-fold at $48 \mathrm{~h}(\mathrm{P}<0.05)$ in hypoxia. Similarly, as shown in Fig. 1D, the in vitro invasion ability of HepG2 cells was increased $1.68 \pm 0.21$-fold and $2.21 \pm 0.16$-fold respectively at $24 \mathrm{~h}, 48 \mathrm{~h}$ in hypoxic condition $(\mathrm{P}<0.05)$. These results suggest that hypoxic stimulus induces EMT and promotes the invasive phenotype of HepG2 cells. 
A

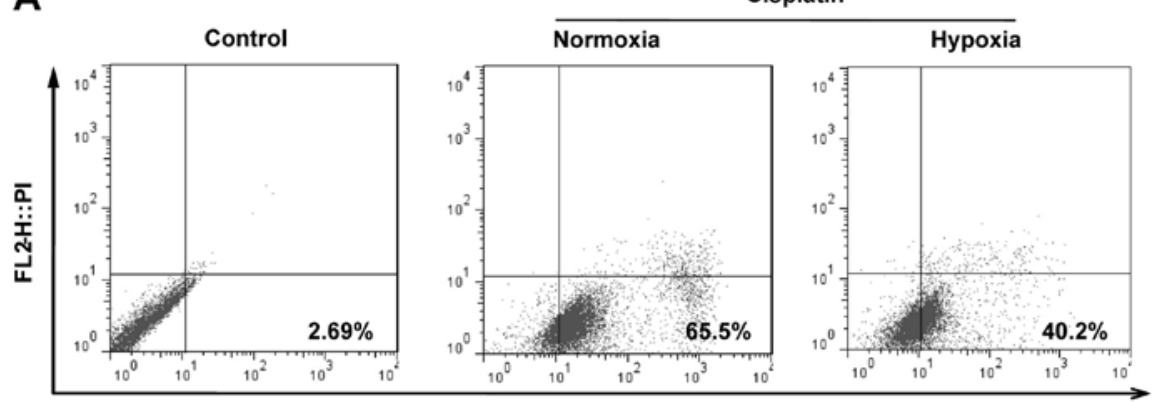

FL1-H:: Annexin VFITC

B

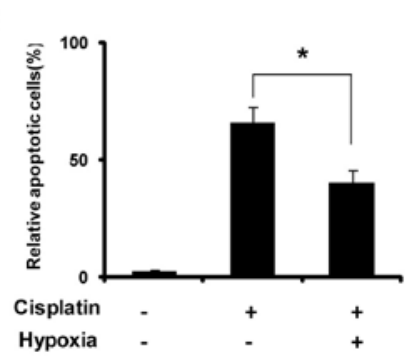

C

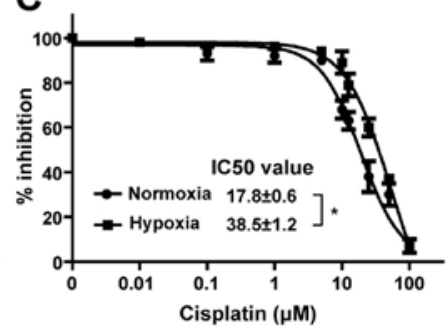

D

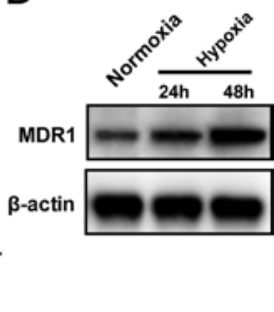

Figure 2. Hypoxia induces cisplatin chemoresistance in HepG2 cells and MDR gene expression under hypoxia. (A) Cells were incubated in normoxia or hypoxia, and then exposed to cisplatin for $48 \mathrm{~h}$, the apoptosis induced by $20 \mu \mathrm{mol} / 1$ cisplatin was determined by Annexin V labeling assays. (B) The percentage of apoptotic cells was plotted (mean \pm SEM in three separate experiments), ${ }^{*} \mathrm{P}<0.05$ cells cultured in hypoxia compared with in normoxia. (C) The cell viability was determined by MTT assay, the $\mathrm{IC}_{50}$ values of $\mathrm{HepG} 2$ cells to cisplatin under normoxic and hypoxic condition were $17.8 \pm 0.6 \mu \mathrm{mol} / 1 \mathrm{and} 38.5 \pm 1.2 \mu \mathrm{mol} / 1$. "P $<0.05$, significant difference between normoxic and hypoxic groups. (D) Expression levels of MDR1 in HepG2 cells under normoxia or hypoxia were examined by Western blotting.
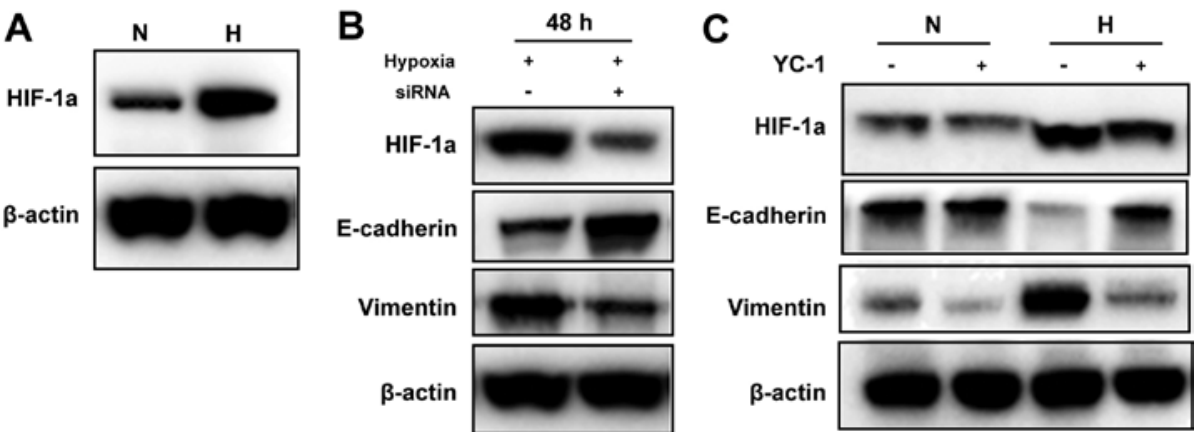

D

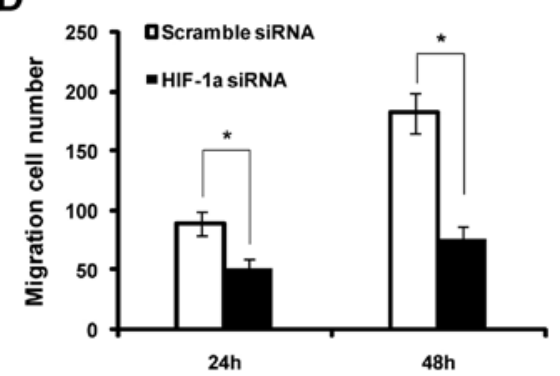

E

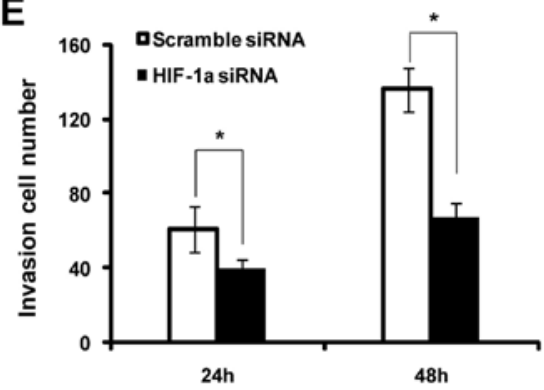

Figure 3. HIF-1 $\alpha$ contributes to EMT induced by hypoxia. (A) After exposure to nomoxia $(\mathrm{N})$ or hypoxia $(\mathrm{H})$ for $48 \mathrm{~h}$, the expression of HIF-1 $\alpha$ was examined by Western blotting. (B) Under hypoxia, HepG2 cells were transfected with HIF-1 $\alpha$ siRNA or scramble siRNA. Forty-eight hours later, the expression of HIF-1 $\alpha$, E-cadherin and vimentin were determined by Western blotting, $\beta$-actin served as loading control. (C) HepG2 cells were treated with $10 \mu \mathrm{mol} / 1$ YC-1 under hypoxia for $48 \mathrm{~h}$. The expression of HIF-1 $\alpha$, E-cadherin and vimentin were examined by Western blotting. (D) Cell migration and invasive ability were analyzed by in vitro migration and invasion assay. The cell number per microscopic field was plotted (mean \pm SEM in three separate experiments). ${ }^{*} \mathrm{P}<0.05$ for cells with HIF-1 $\alpha$ siRNA compared with scramble siRNA.

Hypoxia potentiates chemoresistance to cisplatin in HCC cells. The apoptosis was detected by Annexin V staining. As shown in Fig. $2 \mathrm{~A}$ and $\mathrm{B}$, the results showed that the number of apoptotic cells induced by $20 \mu \mathrm{mol} / \mathrm{l}$ cisplatin was significantly decreased from $65.5 \%$ to $40.2 \%$ under hypoxic condition for $48 \mathrm{~h}(\mathrm{P}<0.05)$. The effect of cisplatin on the growth of HCC cells were also 
A

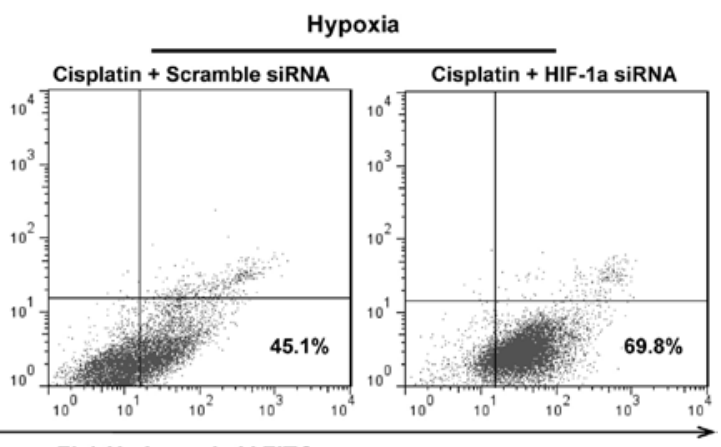

FL1-H::Annexin V FITC

B

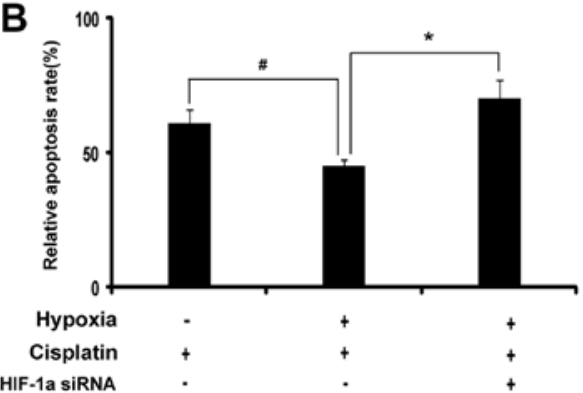

C

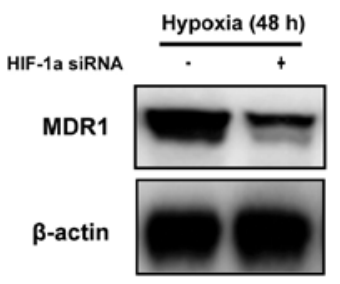

Figure 4. HIF-1 $\alpha$ knockdown enhances apoptosis of hepatocellular carcinoma cells under hypoxia. (A) Cells were transfected with 75 nM HIF-1 $\alpha$ siRNA or scramble siRNA, and then treated with $20 \mu \mathrm{mol} / 1$ cisplatin under nomoxia or hypoxia for $48 \mathrm{~h}$. Apoptosis was determined by Annexin V labeling assays. (B) The percentage of apoptotic cells was plotted (mean \pm SEM in three separate experiments), ${ }^{\prime} \mathrm{P}<0.05$ cells cultured in hypoxia compared to normoxia, ${ }^{*} \mathrm{P}<0.05$ cisplatin with scramble siRNA compared with cisplatin combined with HIF-1 $\alpha$ siRNA. (C) Cells were transfected with HIF-1 $\alpha$ siRNA for 48 h under hypoxia. MDR1 protein was examined by Western blotting.

evaluated by determining cell viability using MTT assay. The $\mathrm{IC}_{50}$ values of cisplatin in HepG2 cells are shown in Fig. 2C. Compared with cells exposed to normoxia, the $\mathrm{IC}_{50}$ value of HepG2 exposed to hypoxia was 2.16-fold higher for cisplatin. Western blotting was used to quantify the expression of MDR1 gene, which is reportedly to be associated with chemoresistance of tumor cells. Indeed, HepG2 cells showed a significant increase of MDR1 expression under hypoxia (Fig. 2D).

HIF-la contributes to EMT and chemoresistance induced by hypoxia. The HIF-1 $\alpha$ is a key mediator of cellular adaptation to hypoxia. To identify whether HIF-1 $\alpha$ plays a critical role during EMT and chemoresistance induced by hypoxia, we performed knockdown or blockage of HIF-1 $\alpha$ expression in hypoxic condition by using $75 \mathrm{nM}$ HIF- $1 \alpha$ siRNA or $10 \mu \mathrm{mol} / 1 \mathrm{YC}-1$ (an inhibitor against HIF-1 $\alpha$ ), and investigated the reversal of EMT and the sensitivity to cisplatin of HepG2 cells under hypoxia. As shown in Fig. 3A, HIF-1 $\alpha$ expression significantly increased under hypoxia compared with normoxia. Under hypoxic condition, HIF-1 $\alpha$ expression significantly decreased by HIF-1 $\alpha$ siRNA at $48 \mathrm{~h}$ after transient transfection, as shown in Fig. 3B. E-cadherin expression increased and vimentin expression decreased following downregulation of HIF-1 $\alpha$. Similarly, E-cadherin expression increased and vimentin expression decreased when HepG 2 cells were treated with $10 \mu \mathrm{mol} / 1 \mathrm{YC}-1$ for $48 \mathrm{~h}$ under normoxia and hypoxia (Fig. 3C). As shown in Fig. 3D and E, the stimulatory effect of hypoxia for HepG2 cell migration and invasion was abolished by knockdown of HIF-1 $\alpha$. Furthermore, we also found that knockdown of HIF-1 $\alpha$ by HIF-1 $\alpha$ siRNA led to a remarkable increase in sensitivity to cisplatin of HepG2 cells. Such treatment in hypoxia provided a synergistic effect of HIF-1 $\alpha$ inhibition and cisplatin, leading to a $24.7 \%$ increase in cell apoptosis rate (Fig. 4A and B). At the same time, we found that under hypoxic condition, the depletion of HIF- $1 \alpha$ by HIF-1 $\alpha$ siRNA significantly decreased the expression of MDR1 (Fig. 4C). Those data showed that HIF-1 $\alpha$ may play a prominent role in cell invasion and chemoresistance to cisplatin by induction of EMT and MDR1 gene expression under hypoxia.

PI3K/AKT signaling contributes to hypoxia-induced HIF-1 $\alpha$ activation. EMT is a dynamic process triggered by microenvironment stimuli. Several cell signaling factors have been implicated in EMT, including PI3K/Akt, $\beta$-catenin and MAPK $(15,18-20)$. Here, we investigated the role of PI3K/Akt in hypoxia-induced EMT. As shown in Fig. 5A, when HepG2 cells were cultured in hypoxia for $48 \mathrm{~h}$, the level of phosphorylated Akt (p-Akt) was substantially increased. At the same time, HIF-1 $\alpha$ expression was increased at the protein level. This indicated that hypoxia increased both HIF-1 $\alpha$ and p-Akt expression levels. Furthermore, we examined whether PI3K/Akt signaling pathway is responsible for hypoxia-induced increase of HIF-1 $\alpha$ in HepG 2 cells. The cells were treated with $10 \mu \mathrm{mol} / 1 \mathrm{LY} 294002$ (a PI3K inhibitor) for $48 \mathrm{~h}$ under hypoxia. The result showed that LY294002 remarkably inhibited the increase of HIF-1 $\alpha$ in HepG2 cells cultured in hypoxic condition (Fig. 5C). These data clearly indicated that PI3K/Akt signaling is an upstream regulator of HIF-1 $\alpha$ in HepG2 cells under hypoxic condition.

Inhibition of PI3K/AKT/HIF-1 $\alpha$ enhanced the chemotherapy efficacy of cisplatin in vivo. To explore whether blockade of HIF-1 $\alpha$ activity enhanced cisplatin-induced cytotoxicity in vivo, we employed an HCC subcutaneous xenograft model. A notable smaller tumor volume and prolongation of animal survival were 
A

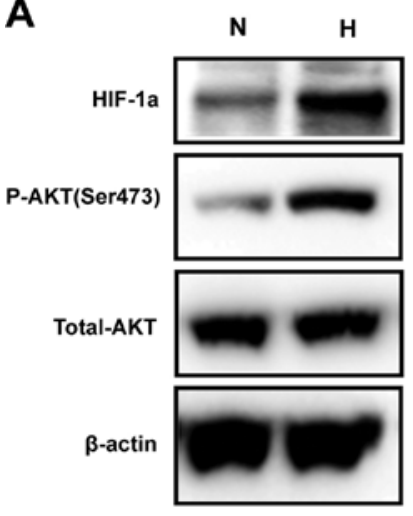

C

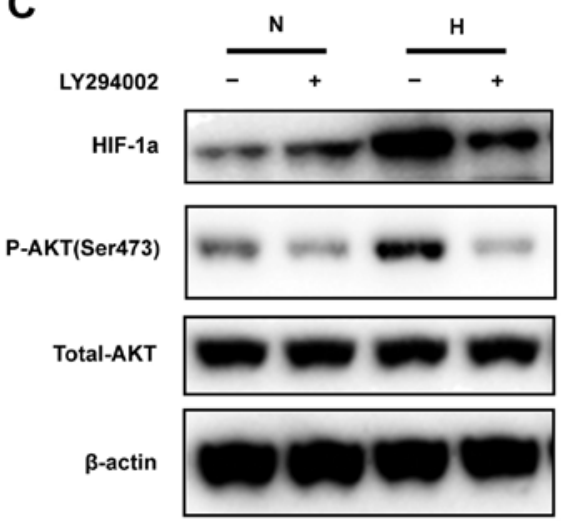

B

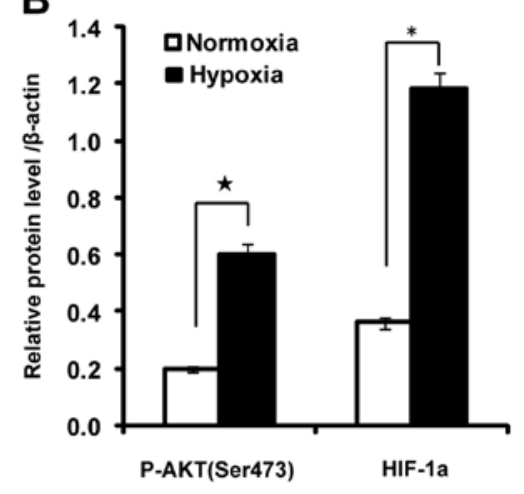

D
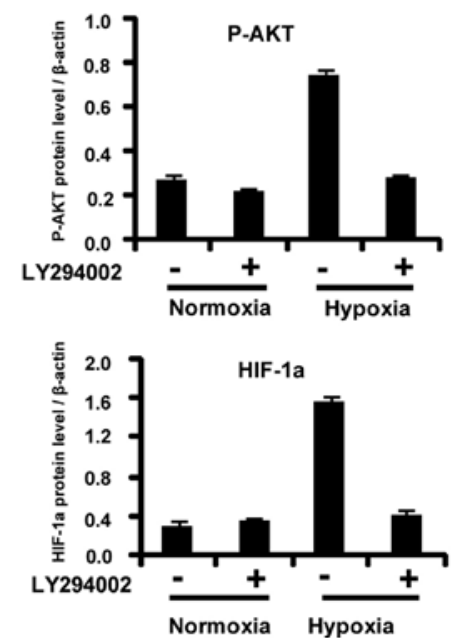

Figure 5. Hypoxia induces HIF-1 $\alpha$ expression through the activation of PI3K/AKT signaling. (A) Cells were cultured in normoxia (N) or hypoxia $(\mathrm{H})$ for $48 \mathrm{~h}$. The expression of phosphorylated Akt (p-Akt, Ser473), total Akt, HIF-1 $\alpha$ and $\beta$-actin were examined by Western blotting. (B) Semi-quantitative analysis of protein level was plotted (mean \pm SEM in three separate experiments). $\beta$-actin served as control. (C) HepG2 cells were treated with $10 \mu \mathrm{mol} / 1$ of the PI3K inhibitor LY294002 or vehicle for $48 \mathrm{~h}$ under normoxia or hypoxia. The expression of p-Akt, HIF-1 $\alpha$ and $\beta$-actin were examined by Western blotting. (D) Semi-quantitative analysis of protein level was plotted (mean \pm SEM in three separate experiments). $\beta$-actin served as control.

observed in the two groups treated with both cisplatin and YC1 or LY294002, respectively. At 21 days, the mean tumor volume was $625.2 \pm 159.3 \mathrm{~mm}^{3}$ in the cisplatin group $(\mathrm{n}=8)$, significantly smaller than that in the control group $\left(1180.2 \pm 105.2 \mathrm{~mm}^{3}\right.$; $\mathrm{P}<0.05)$. Furthermore, cisplatin combined with YC-1 $(\mathrm{n}=8)$ and LY294002 $(n=8)$ treatment produced reductions in tumor volume, which were $312.6 \pm 38.3 \mathrm{~mm}^{3}$ and $280.2 \pm 45.9 \mathrm{~mm}^{3}$, respectively, a significant antitumor effect compared with single treatment with cisplatin or with control $(\mathrm{P}<0.05$; Fig. $6 \mathrm{~A}$ and $\mathrm{B}$ ). The median survival time of the sham control group was 28.0 days and cisplatin alone group was 45.5 days, while cisplatin combined with YC-1 or LY294002 prolonged animal survival, producing a median survival time of 70.0 and 66.5 days, respectively (Fig. 6C). These results indicated such treatment in mice with pre-existing HepG2 tumors provided a synergistic effect of cisplatin and PI3K/AKT and HIF-1 $\alpha$ inhibition, leading to enhanced inhibition of tumor growth and increase of the survival rate.

\section{Discussion}

Hypoxia generally develops in solid tumors, because of rapid cell proliferation that exceed the rate of blood vessel formation. The increase in tumor mass results in aberrant vasculature formation, which reduces the blood supply (21). Cellular adaptations to hypoxia include changes in cell signaling and gene expression, which promote not only cell survival, but also cell invasion and resistance to chemotherapy (22). Therefore, it is extremely important to clarify the comprehensive effects of hypoxia on cancer cell physiology; meanwhile, it is also important to identify specific signaling molecules that can be targeted to eliminate the adverse effects of hypoxia in cancer. Here, we showed that under hypoxic condition, PI3K/Akt-mediated HIF-1 $\alpha$ expression plays a critical role in hypoxia-induced EMT, migration, invasion, and chemoresistance in HCC cells. Our study provides insight into the malignant progression triggered by hypoxic microenvironment in HCC cells.

Firstly, our study indicates that HIF- $1 \alpha$-induced EMT is critical for hypoxia-initiated cell migration and invasion in HCC. EMT, a process vital for morphogenesis during embryonic development, was first recognized as a feature of embryogenesis in the early 1980s $(12,23)$. Currently, it has been generally accepted that EMT plays a critical role in a number of pathological states including cancer metastasis and chronic degenerative fibrosis (24-26). Increasing evidence has shown that EMT promotes tumor progression and metastasis in many solid tumors, in which an important feature is hypoxia. In ovarian carcinoma, breast cancer and renal cell carcinoma, hypoxia induced EMT, 
A

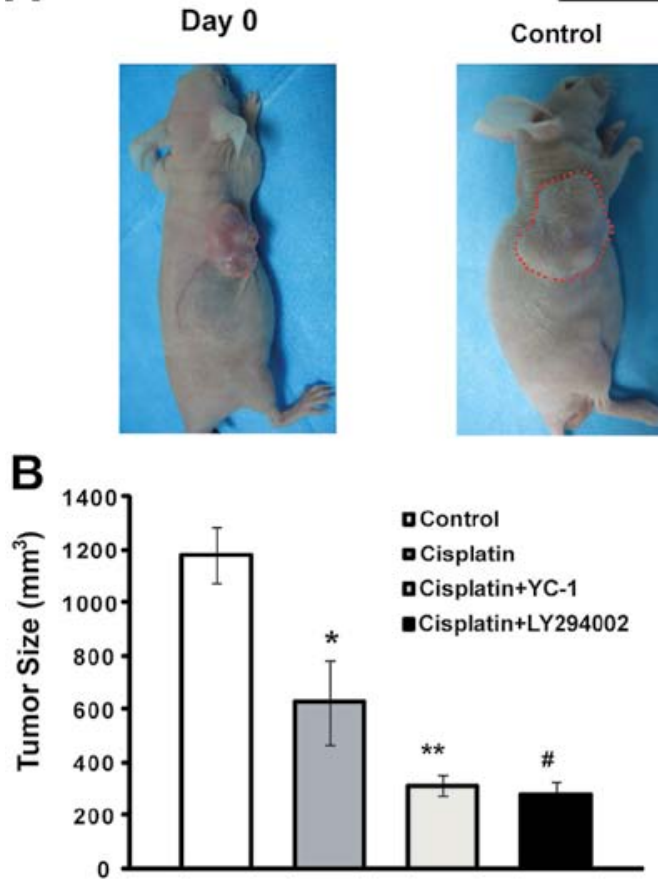

Day 21
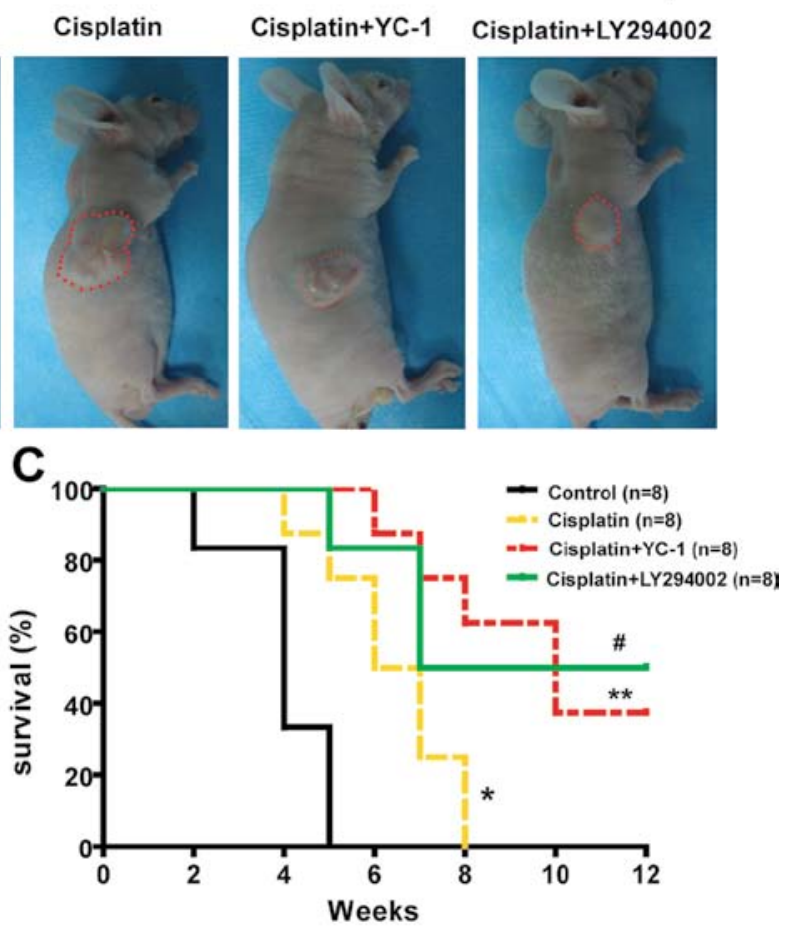

Figure 6. Combination of cisplatin and PI3K/AKT/HIF-1 $\alpha$ inhibition remarkably inhibited the growth of HepG2 xenografts in athymic nude mice. Cells $\left(1 \mathrm{x} 10^{6}\right)$ were injected subcutaneously into the flanks of nude mice. After the tumors reached about $200 \mathrm{~mm}^{3}$ in size, mice received an intraperitoneal injection of cisplatin (4 mg/kg), cisplatin+YC-1 (30 mg/kg) or cisplatin+LY294002 (75 mg/kg), twice per week. (A) At 21 days post-treatment with drugs, representative photographs of tumors of the different treatment groups are shown. (B) Tumor volume was measured at 21 days after the initiation of drugs treatment. ${ }^{*}<0.05$ when compared with control group; ${ }^{* *} \mathrm{P}<0.05,{ }^{\#} \mathrm{P}<0.05$ when compared with single cisplatin group. (C) Kaplan-Meier curve was used to analyze survival difference among different treatment groups $(\mathrm{n}=8)$. ${ }^{*} \mathrm{P}<0.05$ when compared with the sham control group; ${ }^{* *} \mathrm{P}<0.05,{ }^{\#} \mathrm{P}<0.05$ when compared with a single cisplatin group.

which in turn speed the malignant progression $(14,15,27)$. In this study, we demonstrated that hypoxia induces EMT in HCC cells. It is known that HIF-1 $\alpha$ is a critical regulator of cellular and systemic responses to low oxygen levels (28). Our results further confirm the essential role of HIF-1 $\alpha$ to induce EMT under hypoxic microenvironment. For the unresectable HCC, transarterial chemoembolization (TACE) is an alternative to conventional therapies (29). However, this treatment induces a hypoxic microenvironment for residual tumor cells, leading to the activation of hypoxia-induced survival signaling, which contribute to the post-treatment metastasis and drug resistance (30). Our study indicates that HIF-1 $\alpha$-induced EMT is critical for hypoxia-initiated cell migration and invasion in $\mathrm{HCC}$, which also provides the experimental evidence for the unfavorable outcome of few patients after TACE treatment.

Secondly, our study also suggests that HIF-1 $\alpha$ mediates hypoxia-induced cisplatin resistance in HCC cells. Tumor cells surviving under hypoxic conditions commonly become resistant to radiotherapy and chemotherapy (31). In vitro studies have shown that amplification and overexpression of MDR gene, such as MDR1, in cancer cells confer high resistance to many chemotherapy drugs, such as vinca alkaloids, anthracyclines, taxanes, and epipodophyllotoxins (32). In this study, blocking hypoxia-inducible pathways by small interfering RNA against HIF-1 $\alpha$ can recover the chemosensitivity of HCC cells, which were formerly resistance to cisplatin owing to hypoxic exposure. Mechanistically, MDR1 expression was regulated by HIF-1 $\alpha$ under hypoxia. This suggests that HIF-1 $\alpha$-mediated MDR expression is one possible mechanism for cisplatin resistance in HCC cells under hypoxia.

Thirdly, our study reveals a mechanism of HIF-1 $\alpha$ regulation by PI3K/Akt-dependent cell signaling in the process of hypoxia-induced EMT and chemoresistance in HCC cells. Akt could induce HIF-1 $\alpha$ transcriptional activity, leading to HIF-1 $\alpha$ translocation to the nucleus, and then triggered the cascade for survival and progression $(33,34)$. In the present study, we found that hypoxia induced EMT is accompanied by the activation of PI3K/Akt signaling pathway. Inhibition of PI3K by LY294002 decreases HIF-1 $\alpha$ expression. Similar to our finding, one recent report showed that the Akt activation increases the expression of HIF-1 $\alpha$ in colorectal carcinoma (35). Collectively, our study provides evidence that the PI3K/Akt/HIF-1 $\alpha$ pathway is important in promoting $\mathrm{HCC}$ malignant progression and protecting HCC cells from the cytotoxic effects of cisplatin under hypoxic conditions. Consistently, inhibition of this pathway activity enhanced the therapeutic efficacy of chemotherapy and extended the survival in a mouse HCC model.

In summary, our data suggest that PI3K/Akt and HIF-1 $\alpha$ activation after hypoxia plays an essential role in hypoxia-induced EMT and drug resistance, leading to unfavorable treatment outcome. Therefore, a combination strategy of chemotherapy with PI3K/Akt and HIF-1 $\alpha$ blockade will shed light on the enhancement of therapeutic efficacy of chemotherapy for HCC treatment. Our study not only reveals a critical mechanism 
underlying hypoxia-induced metastasis and chemoresistance but also provides important implications in the development of novel treatment strategies for metastatic cancers.

\section{Acknowledgements}

This study was supported by National Natural Science Foundation of China grant (No. 30801152).

\section{References}

1. McGlynn KA and London WT: The global epidemiology of hepatocellular carcinoma: present and future. Clin Liver Dis 15: 223-243, 2011.

2. Jemal A, Bray F, Center MM, Ferlay J, Ward E and Forman D: Global cancer statistics. CA Cancer J Clin 61: 69-90, 2011.

3. Nordenstedt H, White DL and El-Serag HB: The changing pattern of epidemiology in hepatocellular carcinoma. Dig Liver Dis 42 (Suppl. 3): S206-S214, 2010.

4. Brown JM and Wilson WR: Exploiting tumour hypoxia in cancer treatment. Nat Rev Cancer 4: 437-447, 2004.

5. Giglia JL, Antonia SJ, Berk LB, Bruno S, Dessureault S and Finkelstein SE: Systemic therapy for advanced hepatocellular carcinoma: past, present, and future. Cancer Control 17: 120-129, 2010.

6. Chan DA and Giaccia AJ: Hypoxia, gene expression, and metastasis. Cancer Metastasis Rev 26: 333-339, 2007.

7. Denko NC, Fontana LA, Hudson KM, et al: Investigating hypoxic tumor physiology through gene expression patterns. Oncogene 22: 5907-5914, 2003.

8. Farazi PA and DePinho RA: Hepatocellular carcinoma pathogenesis: from genes to environment. Nat Rev Cancer 6: 674-687, 2006.

9. Chang Q, Jurisica I, Do T and Hedley DW: Hypoxia predicts aggressive growth and spontaneous metastasis formation from orthotopically grown primary xenografts of human pancreatic cancer. Cancer Res 71: 3110-3120, 2011.

10. Pennacchietti S, Michieli P, Galluzzo M, Mazzone M, Giordano S and Comoglio PM: Hypoxia promotes invasive growth by transcriptional activation of the met protooncogene. Cancer Cell 3 : 347-361, 2003

11. Brahimi-Horn MC, Chiche $\mathrm{J}$ and Pouyssegur J: Hypoxia and cancer. J Mol Med 85: 1301-1307, 2007.

12. Thiery JP: Epithelial-mesenchymal transitions in development and pathologies. Curr Opin Cell Biol 15: 740-746, 2003.

13. Jou J and Diehl AM: Epithelial-mesenchymal transitions and hepatocarcinogenesis. J Clin Invest 120: 1031-1034, 2010.

14. Imai $\mathrm{T}$, Horiuchi $\mathrm{A}$, Wang $\mathrm{C}$, et al: Hypoxia attenuates the expression of E-cadherin via up-regulation of SNAIL in ovarian carcinoma cells. Am J Pathol 163: 1437-1447, 2003.

15. Lester RD, Jo M, Montel V, Takimoto S and Gonias SL: uPAR induces epithelial-mesenchymal transition in hypoxic breast cancer cells. J Cell Biol 178: 425-436, 2007.

16. Sullivan R, Pare GC, Frederiksen LJ, Semenza GL and Graham CH: Hypoxia-induced resistance to anticancer drugs is associated with decreased senescence and requires hypoxia-inducible factor-1 activity. Mol Cancer Ther 7: 1961-1973, 2008.
17. Comerford KM, Wallace TJ,Karhausen J,Louis NA, Montalto MC and Colgan SP: Hypoxia-inducible factor-1-dependent regulation of the multidrug resistance (MDR1) gene. Cancer Res 62: 3387-3394, 2002.

18. Larue L and Bellacosa A: Epithelial-mesenchymal transition in development and cancer: role of phosphatidylinositol 3' kinase/ AKT pathways. Oncogene 24: 7443-7454, 2005.

19. Thiery JP and Sleeman JP: Complex networks orchestrate epithelial-mesenchymal transitions. Nat Rev Mol Cell Biol 7: 131-142, 2006.

20. Liu L, Zhu XD, Wang WQ, et al: Activation of beta-catenin by hypoxia in hepatocellular carcinoma contributes to enhanced metastatic potential and poor prognosis. Clin Cancer Res 16: 2740-2750, 2010

21. O'Reilly MS, Holmgren L, Chen C and Folkman J: Angiostatin induces and sustains dormancy of human primary tumors in mice. Nat Med 2: 689-692, 1996.

22. Zhou J, Schmid T, Schnitzer S and Brune B: Tumor hypoxia and cancer progression. Cancer Lett 237: 10-21, 2006.

23. Thiery JP: Epithelial-mesenchymal transitions in tumour progression. Nat Rev Cancer 2: 442-454, 2002.

24. Wu Y and Zhou BP: New insights of epithelial-mesenchymal transition in cancer metastasis. Acta Biochim Biophys Sin (Shanghai) 40: 643-650, 2008.

25. Kalluri R and Neilson EG: Epithelial-mesenchymal transition and its implications for fibrosis. J Clin Invest 112: 1776-1784, 2003.

26. Rastaldi MP: Epithelial-mesenchymal transition and its implications for the development of renal tubulointerstitial fibrosis. J Nephrol 19: 407-412, 2006.

27. Krishnamachary B, Zagzag D, Nagasawa H, et al: Hypoxiainducible factor-1-dependent repression of E-cadherin in von Hippel-Lindau tumor suppressor-null renal cell carcinoma mediated by TCF3, ZFHX1A, and ZFHX1B. Cancer Res 66: 2725-2731, 2006.

28. Pouyssegur J, Dayan F and Mazure NM: Hypoxia signalling in cancer and approaches to enforce tumour regression. Nature 441: 437-443, 2006

29. Grieco A, Marcoccia S, Miele L, et al: Transarterial chemoembolization (TACE) for unresectable hepatocellular carcinoma incirrhotics: functional hepaticreserveand survival.Hepatogastroenterology 50: 207-212, 2003

30. Llovet JM, Real MI, Montana X, et al: Arterial embolisation or chemoembolisation versus symptomatic treatment in patients with unresectable hepatocellular carcinoma: a randomised controlled trial. Lancet 359: 1734-1739, 2002.

31. Brown JM: The hypoxic cell: a target for selective cancer therapyeighteenth Bruce F. Cain Memorial Award lecture. Cancer Res 59: 5863-5870, 1999.

32. Scala S, Akhmed N, Rao US, et al: P-glycoprotein substrates and antagonists cluster into two distinct groups. Mol Pharmacol 51: 1024-1033, 1997.

33. Jiang BH and Liu LZ: AKT signaling in regulating angiogenesis. Curr Cancer Drug Targets 8: 19-26, 2008.

34. Semenza GL: Targeting HIF-1 for cancer therapy. Nat Rev Cancer 3: 721-732, 2003

35. Pez F, Dayan F, Durivault J, et al: The HIF-1-inducible lysyl oxidase activates HIF-1 via the Akt pathway in a positive regulation loop and synergizes with HIF-1 in promoting tumor cell growth. Cancer Res 71: 1647-1657, 2011. 